The Astrophysical Journal, 646:1038-1042, 2006 August 1

(C) 2006. The American Astronomical Society. All rights reserved. Printed in U.S.A.

\title{
SPITZER MIPS LIMITS ON ASTEROIDAL DUST IN THE PULSAR PLANETARY SYSTEM PSR B1257+12
}

\author{
G. Bryden, ${ }^{1}$ C. A. Beichman, ${ }^{2}$ G. H. Rieke, ${ }^{3}$ J. A. Stansberry, ${ }^{3}$ K. R. Stapelfeldt, ${ }^{1}$ \\ D. E. Trilling, ${ }^{3}$ N. J. Turner, ${ }^{1}$ and A. Wolszczan ${ }^{4}$ \\ Received 2006 January 30; accepted 2006 April 5
}

\begin{abstract}
With the MIPS camera on Spitzer, we have searched for far-infrared emission from dust in the planetary system orbiting pulsar PSR B1257+12. With accuracies of $0.05 \mathrm{mJy}$ at $24 \mu \mathrm{m}$ and $1.5 \mathrm{mJy}$ at $70 \mu \mathrm{m}$, photometric measurements find no evidence for emission at these wavelengths. These observations place new upper limits on the luminosity of dust with temperatures between 20 and $1000 \mathrm{~K}$. They are particularly sensitive to dust temperatures of 100-200 K, for which they limit the dust luminosity to below $3 \times 10^{-5}$ of the pulsar's spin-down luminosity, 3 orders of magnitude better than previous limits. Despite these improved constraints on dust emission, an asteroid belt similar to the solar system's cannot be ruled out.
\end{abstract}

Subject headings: circumstellar matter — infrared: stars — pulsars: individual (PSR B1257+12)

\section{INTRODUCTION}

Before the discovery of extrasolar planets around mainsequence stars (Mayor \& Queloz 1995; Marcy \& Butler 1995), pulsar timing measurements provided the first evidence for an extrasolar planetary system (Wolszczan \& Frail 1992). The initial discovery of two planets was later expanded to three: $0.02 \pm$ $0.002 M_{\oplus}$ at $0.19 \mathrm{AU}, 4.3 \pm 0.2 M_{\oplus}$ at $0.36 \mathrm{AU}$, and $3.9 \pm$ $0.2 M_{\oplus}$ at 0.46 AU (Wolszczan 1994; Wolszczan et al. 2000; Konacki \& Wolszczan 2003). Orbital analysis of the pulsar timing measurements reveals a coplanar system with the outer planets in 3:2 orbital resonance (Konacki \& Wolszczan 2003), strongly suggesting that their formation mechanism must involve a preplanetary disk of material circling the neutron star. A variety of theories have been proposed for the formation of such a disk in this system (see Miller \& Hamilton 2001): from the remains of a merger event (Podsiadlowski et al. 1991), from the disruption or ablation of a stellar companion (Stevens et al. 1992; Tavani \& Brookshaw 1992), or from the fallback of supernova ejecta (Lin et al. 1991). The presence of an early disk is further motivated by theories for millisecond pulsar formation, which generally use an accretion disk to spin up the pulsar (Michel \& Dessler 1985).

In its presumed disk origin, the pulsar system's history is thought to be similar to our own solar system and its protostellar nebula. Evidence for such disks around hydrogen-burning stars is clear. Massive protoplanetary disks are commonly found in young star-forming regions, both inferred from spectral energy distributions (Beckwith et al. 1990) and seen directly in silhouette (McCaughrean \& O’Dell 1996). The older remnants of these disks were first discovered around main-sequence stars by the Infrared Astronomical Satellite (IRAS; Aumann et al. 1984), with many debris disks later identified by the Infrared Space Observatory (ISO; Habing et al. 2001) and now with Spitzer (Rieke et al. 2005). Dusty disks are frequently found around main-sequence stars over a range of spectral types and ages, yet no disk emission has ever been detected from around a pulsar.

\footnotetext{
1 Jet Propulsion Lab, 4800 Oak Grove Drive, Pasadena, CA 91109.

2 Michelson Science Center, California Institute of Technology, Pasadena, CA 91125.

3 Steward Observatory, University of Arizona, 933 North Cherry Avenue, Tucson, AZ 85721.

4 Department of Astronomy and Astrophysics, Pennsylvania State University, University Park, PA 16802.
}

Many attempts have been made to survey nearby pulsars for dust emission. The broadest of these surveys, an examination of the positions of 478 pulsars in the IRAS Point Source Catalog, failed to identify any excess beyond that expected from coincidental alignment (van Buren \& Terebey 1993). Many smaller but more sensitive surveys of pulsars have been made, both in the infrared (IR; Foster \& Fischer 1996; Koch-Miramond et al. 2002; Lazio \& Fischer 2004) and at submillimeter wavelengths (Phillips \& Chandler 1994; Greaves \& Holland 2000; Löhmer et al. 2004). As the host to a planetary system, PSR B1257+12 has been targeted in particular, both within surveys and by other specific observations. A range of wavelengths has been considered, from visible (Abazajian et al. 2005) to near-IR, ${ }^{5}$ mid-IR (Foster \& Fischer 1996), far-IR (Moshir et al. 1990), and submillimeter (Phillips \& Chandler 1994; Greaves \& Holland 2000; Löhmer et al. 2004). (A summary of these PSR B1257+12 observations is shown in Fig. 1 below.) In each case, only upper limits for dust emission were obtained.

Nevertheless, these searches have been limited by their sensitivity, particularly at far-IR wavelengths. Very high upper limits for the dust mass $\left(\sim 100 M_{\oplus}\right)$ are commonly cited (Löhmer et al. 2004; Lazio \& Fischer 2004). Pulsars tend to be much farther away than the main-sequence stars identified as having debris disks (typically tens of parsecs for solar-type stars; Bryden et al. 2006), making detection more difficult. Perhaps more importantly, the pulsar's efficiency in heating any dust that might be present may be much lower than for hydrogen-burning stars, whose radiation peaks in visible light (see $\S 3$ below). Given the difficulty in detecting dust around pulsars, Spitzer, with unprecedented sensitivity to IR radiation, is an ideal observatory for continuing the ongoing search for dust in the PSR B1257+12 system. Below, we describe such Spitzer observations (§ 2) and then use them to place stricter limits on the PSR B1257+12 dust luminosity $(\S 3)$.

\section{OBSERVATIONS}

PSR B1257+12 was observed with the Spitzer long-wavelength camera, MIPS, on 2005 June 21 at both 24 and $70 \mu \mathrm{m}$. Our overall data analysis is similar to that previously described by Beichman et al. (2005a), Bryden et al. (2006), and T. N. Gautier et al. (2006,

\footnotetext{
5 Vizier Online Data Catalog, 2246 (Cutri, R. M., et al., 2003).
} 


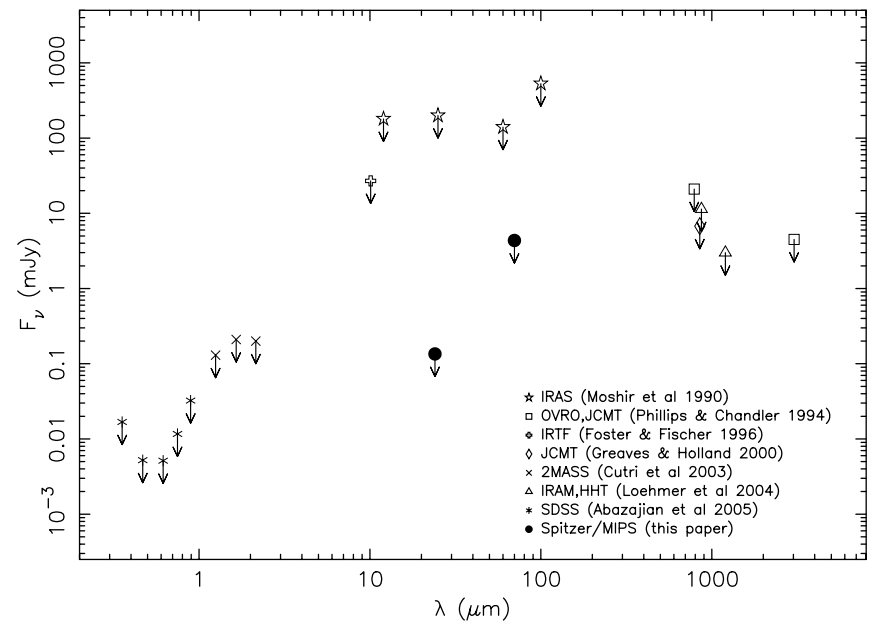

FIG. 1.-Observational upper limits for emission from the PSR B1257+12 system. Over a range of wavelengths from visible to $\mathrm{mm}, 3 \sigma$ upper limits are shown for data from various sources (see legend). Our MIPS 24 and $70 \mu \mathrm{m}$ results are shown as filled circles.

in preparation). At $24 \mu \mathrm{m}$, mosaicked images were created from the raw data using the DAT software developed by the MIPS instrument team (Gordon et al. 2005). Several additional corrections were applied, including the subtraction of smooth gradients across the field (to remove scattered light effects parallel to the scan mirror direction) and the application of a second-order flat, derived from the data (to correct for dark latents, residual jail bars, and broad flat-field trends). At $70 \mu \mathrm{m}$, images were processed beyond the standard DAT software to correct for time-dependent transients, corrections that can greatly improve the sensitivity of the measurements (Gordon et al. 2004). For both wavelengths, aperture photometry was performed using calibration factors, apertures sizes, background annuli, and aperture corrections as in Beichman et al. (2005a). PSR B1257+12 was not observed at $160 \mu \mathrm{m}$, due to the high background noise in MIPS images at that wavelength (typically tens of millijanskys) relative to existing submillimeter limits.

At the observed wavelengths, we fail to detect significant emission from the pulsar system. Upper limits are calculated directly from the noise levels measured within each field. At $24 \mu \mathrm{m}$, where we have integrated over 5 cycles of $3 \mathrm{~s}$ exposures, we achieve a $(1 \sigma)$ sensitivity of $0.045 \mathrm{mJy}$. At $70 \mu \mathrm{m}$, with 5 cycles of $10 \mathrm{~s}$ exposures, our sensitivity is $1.45 \mathrm{mJy}$. This level of accuracy at $70 \mu \mathrm{m}$ is better than that typically seen in similar observations (e.g., Beichman et al. 2005a), a reflection of the fortuitously low background level in this field. The accuracy of this observation is likely to be limited by confusion with background extragalactic sources, such that longer integration time would not significantly improve the results (Dole et al. 2004; Bryden et al. 2006).

The $3 \sigma$ upper limits calculated here are shown in Figure 1 alongside those from previous investigations. While $\sim$ mJy limits have been obtained at near-IR and submillimeter wavelengths, previous observations have been least sensitive at the far-IR wavelengths most commonly used to detect dust emission around mainsequence stars. At $24 \mu \mathrm{m}$, the Spitzer limit is more than 3 orders of magnitude better than IRAS, while at $70 \mu \mathrm{m}$ there is a factor of $\sim 40$ improvement. The following section translates these limits on flux into constraints on the dust temperature and luminosity.

\section{DUST CONSTRAINTS}

Other searches for dust around PSR B1257+12 have generally compared their observational limits with the dust model of
Foster \& Fischer (1996). In this model, the dust absorbs and reradiates some fraction of the energy that the pulsar is known to be losing as it spins down. This spin-down luminosity, $L_{\mathrm{SD}}=$ $4 \pi^{2} I \dot{P} / P^{3}$, can be easily calculated from the pulsar's period, $P$, and spin-down rate, $\dot{P}$. The pulsar's moment of inertia, $I$, is assumed to be $10^{45} \mathrm{~g} \mathrm{~cm}^{2}$ (Foster \& Fischer 1996). For PSR B1257+12 $\left(P=6.2 \mathrm{~ms}, \dot{P}=1.1 \times 10^{-16} \mathrm{~ms} \mathrm{~s}^{-1}\right.$; Konacki \& Wolszczan 2003) the spin-down luminosity is $5.2 L_{\odot}$. Following Foster \& Fischer (1996), previous work (e.g., Greaves \& Holland 2000; Löhmer et al. 2004) typically assumed a dust luminosity of $1 \%$ of $L_{\mathrm{SD}}$, a plausible upper limit for the fraction of energy a thick dust disk might intercept.

Rather than assume an ad hoc dust luminosity, we instead choose to treat $L_{\text {dust }} / L_{\mathrm{SD}}$ as the primary unknown quantity whose value we can place limits on. In fact, the dust luminosity can be directly constrained from flux measurements, depending on the temperature of the dust (e.g., Beichman et al. 2005a). The temperature, however, is very uncertain. The standard Foster \& Fischer (1996) dust models assign temperatures of 10-20 K to the dust, but the physical mechanism for converting the pulsar's rotational energy into thermal dust emission is not specified. The dust temperature in these models is essentially arbitrary. The ability of the various-sized dust grains to absorb the pulsar's emitted energy is not considered, nor is the distance of the dust from that energy source.

Around solar-type stars, which emit radiation at wavelengths readily absorbed by micron-sized dust grains, dust at 1 AU can reach temperatures of several hundred K. Around pulsars with solar-like luminosities spread over a range of wavelengths, the absorption is presumably less efficient, resulting in lower temperatures at the same orbital distances. Should a belt of material circle outside the orbits of the PSR B1257+12 planets, temperatures of $\sim 50-100 \mathrm{~K}$ might be expected, depending on the pulsar's spectral distribution. The fraction of PSR B1257+12's luminosity emitted at various wavelengths is directly limited by observations. Although the pulsar emission model of Malov (2003) predicts a high X-ray flux $\left(5 \times 10^{-9} \mathrm{ergs}^{-2} \mathrm{~s}^{-1}\right)$, the lack of detection by Chandra sets an upper limit of $6 \times 10^{-15} \mathrm{ergs} \mathrm{cm}^{-2} \mathrm{~s}^{-1}$ (G. G. Pavlov et al. 2006, in preparation), less than $10^{-6}$ of the spin-down luminosity. Optical nondetections (e.g., Abazajian et al. 2005) provide similarly strict limits for visible emission. Instead, most of the pulsar's spin-down energy is emitted as relativistic particles (Gaensler \& Slane 2006). In this case, the heating of small dust grains is minimal; each particle impact with a dust particle will eject a nuclide from the system without imparting kinetic energy into the parent dust grain. The heating efficiency for the dust in a thin disk may then be very low, with correspondingly low temperatures. Rocks that are larger than the stopping depth for the relativistic particles $(\sim 100 \mathrm{~cm}$; Miller \& Hamilton 2001), however, will absorb most of the incoming energy and will be much more efficient at converting the pulsar's spin-down energy into thermal radiation.

Unlike the highly beamed radio emission, pulsars' particle winds are fairly symmetric, as evidenced by the near-spherical shapes of pulsar wind nebulae (e.g., Kennel \& Coroniti 1984; Gaensler \& Slane 2006). A key uncertainty, however, is the location where the pulsar's magnetic dipole radiation transitions into a relativistic particle wind. Close to the pulsar, at its light cylinder radius $(c / \Omega=300 \mathrm{~km})$, the outward flow is thought to be dominated by the Poynting flux $(\boldsymbol{E} \times \boldsymbol{B}$; Gaensler \& Slane 2006). Observations of pulsar nebulae, however, find that the flux on larger scales is concentrated in a particle wind. For the Crab Pulsar's nebula, for example, modeling of the energy deposition into the wind termination shock requires that a large 


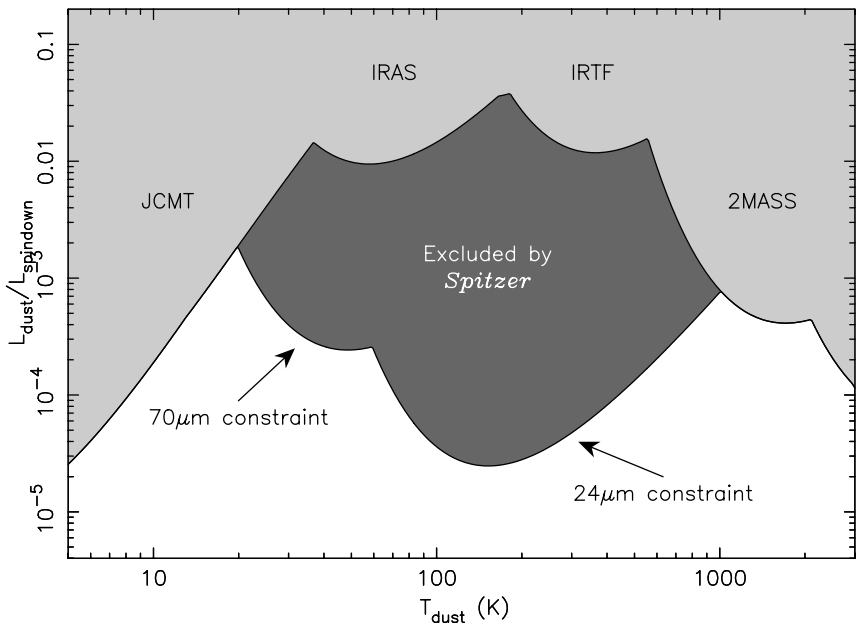

FIG. 2.- Limits on the temperature and luminosity of dust in the PSR $\mathrm{B} 1257+12$ system. The light shaded region is based on previous observations, while the dark shaded region represents dust parameters ruled out by the observations described here. Dust emissivity is assumed to fall off linearly as the wavelength increases past $100 \mu \mathrm{m}$. To convert fluxes to luminosities, we adopt a pulsar distance of $450 \mathrm{pc}$ based on the Taylor \& Cordes (1993) model for the Galactic electron distribution, recently updated by Cordes \& Lazio (2002); this distance is uncertain by $\sim 20 \%$.

fraction $(\gtrsim 99 \%)$ of the energy has been converted into a particle wind before reaching a few parsecs from the pulsar (Kennel \& Coroniti 1984; Begelman \& Li 1992). Exactly where or how transition occurs is not known. Assuming that the particle wind has fully developed before reaching $\sim \mathrm{AU}$ orbital distances, the pulsar's spin-down energy will effectively heat all large bodies in the planetary system; otherwise only ionized matter would be strongly influenced.

Given the uncertainty in the dust temperature, we leave it as a free parameter to be constrained by observations. Figure 2 shows our constraints on $L_{\mathrm{dust}} / L_{\mathrm{SD}}$ for a range of dust temperatures, under the assumption that the dust can be characterized by a single dominant value. (In reality some range of temperatures would exist for dust with a range of grain sizes and orbital locations, even more so if stochastic heating is responsible for large temporary increases in dust temperature for a small fraction of the grains.) For each temperature, we calculate the maximum blackbody emission that is consistent with the observed $3 \sigma$ limits. Each of the curved segments in Figure 2 corresponds to an individual observation at a specific wavelength, with the temperatures that an observation is most sensitive to depending on that wavelength. As seen in the figure, the Spitzer observations create more stringent limits on dust luminosity for dust with temperatures between 20 and $1000 \mathrm{~K}$. (Above $1000 \mathrm{~K}$, near-IR photometry is more accurate, while below $20 \mathrm{~K}$ submillimeter measurements are more sensitive.) Better than 2 orders of magnitude improvement in sensitivity is achieved over much of this range, with limits on $L_{\text {dust }}$ as low as $2.5 \times 10^{-5} L_{\mathrm{SD}}$ for $\sim 150 \mathrm{~K}$ dust. For highemissivity dust orbiting at $2.5 \mathrm{AU}$, this corresponds to an upper limit on the emitting area of $5 \times 10^{23} \mathrm{~cm}^{2}$.

\section{DISCUSSION}

Based on our Spitzer observations, we constrain the luminosity of dust emission around PSR B $1257+12$ to be $<10^{-4} L_{\mathrm{SD}}$ for temperatures of $\sim 50$ to $500 \mathrm{~K}$. This limit allows us rule out a dense, thick disk that absorbs and thermalizes a large fraction of the emitted energy - essentially the same general conclusion as reached by previous authors. This nondetection of IR excess, however, does not preclude the presence of an optically thin debris disk orbiting the pulsar. While the Spitzer observations represent a great improvement over previous IR observations, they still do not have an optimal level of accuracy in terms of $L_{\text {dust }} / L_{\mathrm{SD}}$. Observations of nearby main-sequence stars at this sensitivity level would be able to detect only the brightest debris disks. For example, only $\sim 2 \%$ of solar-type stars have luminosities $>10^{-4} L_{\star}$ (Bryden et al. 2006). A much greater fraction of these stars $(\sim 12 \%)$ have disks within an order of magnitude below this limit, while the solar system has $L_{\text {dust }} / L_{\star}$ even lower $\left(\sim 10^{-7}\right.$ to $10^{-6}$ for the Kuiper Belt; Stern 1996). Even if the dust is efficiently heated by the pulsar, there may simply be too little for us to detect.

A belt of planetesimals circling at a few AU, similar to our own asteroid belt, might still be expected. In other systems, the detection of emitting dust outside of the orbits of known extrasolar planets (Beichman et al. 2005a), similar to the Kuiper Belt in our solar system, suggests that an architecture of outer debris belts encircling inner planets may be a general consequence of planet formation. Goździewski et al. (2005), meanwhile, have investigated the long-term stability of test particles orbiting in the PSR B $1257+12$ system, finding that most of the region outside of $1 \mathrm{AU}$ is stable to perturbations from the three inner planets. While high-energy particles emitted from the pulsar should evaporate very small bodies on a relatively short timescale, Miller \& Hamilton (2001) estimate that planetesimals larger than $\sim 1 \mathrm{~km}$ in size can survive the lifetime of the pulsar. (The age of the pulsar is estimated from its spin-down timescale, $P / 2 \dot{P}$, to be $<1$ Gyr.) In fact, there is now evidence for an asteroid-like object orbiting at $2.4 \mathrm{AU}$ with a mass of $4 \times 10^{-4} M_{\oplus}$, about twice the mass of Ceres, the largest asteroid in the solar system (A. Wolszczan \& M. Konacki 2006, in preparation). Ongoing pulsar timing measurements will probe down to even smaller masses and can eventually determine whether many large asteroids are present in this system.

From this pulsar timing, some bounds can be placed on the total mass contained in larger planetesimals. Pulsar timing measurements provide direct constraints on the mass distribution, which is restricted to large asteroid-like mass concentrations. For an extended disk, the total mass potentially contained in asteroids is limited by the requirement that the belt not grind itself away over lifetime of the system. If the age of the pulsar is $\sim 1 \mathrm{Gyr}$, the timescale for destructive collisions between the largest objects must be longer in order for them to survive. A disk with tens of Earth masses of planetesimals would break itself down into smaller objects relatively quickly. The solar system's asteroid belt, by comparison, is evolving on gigayear timescales (Dermott et al. 2002). Similarly, the collisional timescale for a disk of planetesimals a few AU from a pulsar is of order $\sim R_{\mathrm{pl}} M_{\oplus} / R_{\oplus} M_{\text {disk }}$ Gyr, where $R_{\mathrm{pl}}$ is the typical size of the planetesimals and $M_{\text {disk }}$ is their combined mass (see, e.g., Dominik $\&$ Decin 2003), such that a $1 M_{\oplus}$ disk of planetesimals would have ground down already.

The mass contained in smaller bodies is less clear. Given the possibility of weak coupling between dust and the energy emitted by the pulsar, the observational constraints on dust luminosity do not translate well into upper limits on dust mass in the system. Rapid dust removal, however, suggests that the dust mass not be arbitrarily large. If the disk is optically thin toward the pulsar wind, dust particles will be ablated by relativistic particle impacts on timescales of $<1$ yr (Miller \& Hamilton 2001), i.e., even faster than the removal of solar system dust by PoyntingRobertson drag ( $\sim 10^{3}$ yr for micron-sized grains; Gustafson 1994). As in our own system, dust could be continually replenished by a collisional cascade from larger rocks, but the fast removal 
process would limit the amount of dust to a level below that from a similar cascade around a main-sequence star.

In order to assess the effect of this ablation on the IR emission from a pulsar encircling debris disk, we consider a collisional cascade model in which large planetesimals are continuously shattered to produce smaller and smaller objects. The equilibrium slope of the resultant distribution is $d n / d a \propto a^{-3.5}$ (Dohnanyi 1968), such that the smallest grains dominate the overall surface area while the largest bodies comprise the bulk of the system's mass. On top of this standard collisional cascade we have included the important new effect in a pulsar environment - particle ablation by the pulsar wind. Otherwise the simulations we present here are relatively simple compared to detailed asteroid belt models (e.g., Durda \& Dermott 1997). In particular, we assume that (1) the binding strength is independent of object size, (2) impactors can catastrophically disrupt objects up to 10 times their size, and (3) the distribution of debris created in such an impact goes as $d n / d a \propto a^{-3.5}$. While these simplifications neglect some of the physics (for a full discussion and detailed models see Davis et al. 2002), they allow us to calculate illustrative models of the dust surrounding a pulsar.

Before including the effects of the pulsar wind, we start with a standard collisional cascade similar to our own asteroid belt's. Starting with $10 M_{\oplus}$ of $500 \mathrm{~km}$ radius planetesimals located at 2.5 AU, after $1 \mathrm{Gyr}$ of collisions the belt of material has ground down to just $10^{-2} M_{\oplus}$ (cf. $\sim 10^{-3} M_{\oplus}$ contained in the asteroid belt; Davis et al. 2002). The resulting size distribution (dashed line in Fig. 3) extends from the large planetesimals down to small dust. For grain sizes smaller than $\sim 1 \mathrm{~mm}$, Poynting-Robertson drag begins to remove dust faster than it can be replenished by collisions, resulting in a small but significant flattening of the size distribution for the smallest grains. In the solar system, for example, the combination of collisions and Poynting-Robertson drag result in a typical dust radius of several $100 \mu \mathrm{m}$ within the asteroid belt (Dermott et al. 2002) while the interstellar dust particles reaching the Earth have sizes somewhat smaller $(\sim 100 \mu \mathrm{m}$; Love \& Brownlee 1993). The total dust area in our collisional model without ablation is $5 \times 10^{22} \mathrm{~cm}^{2}$. For a given total mass, varying the unknown material composition tends to have relatively little effect on this dust area (denser material has less emitting area per mass, but is more resistant to removal by radiation pressure; in the simple model considered here these effects exactly offset each other).

At the other extreme we also consider the distribution of object sizes under the influence of ablation, but with no collisions (Fig. 3, dotted line). This would apply to a very sparse distribution of planetesimals. As calculated by Miller \& Hamilton (2001), objects up to several $\mathrm{km}$ in size are worn down by the pulsar wind over $1 \mathrm{Gyr}$. The other important size scale for this distribution is $100 \mathrm{~cm}$, the stopping distance for the relativistic particles. For objects larger than this, mass loss is independent of size [giving equal numbers of objects in linear mass bins or $d n / d(\log a) \propto a^{3}$, while below $100 \mathrm{~cm}$ the mass loss is directly proportional to mass [giving equal numbers in logarithmic mass bins or $d n / d(\log a)=$ constant].

When collisions are again considered in addition to the effects of ablation (Fig. 3, solid line ), objects smaller than a kilometer in size are continually resupplied by the disruptive collisions of larger objects. This replenishment greatly increases the amount of small dust and rocks above the simple ablation estimate (compare the dotted and solid lines in Fig. 3). The ability of Poynting-Robertson drag to remove the smallest dust is unclear. (Poynting-Robertson drag is caused by particles absorbing photons with no angular momentum and then reemitting them in the dust's rotating frame.

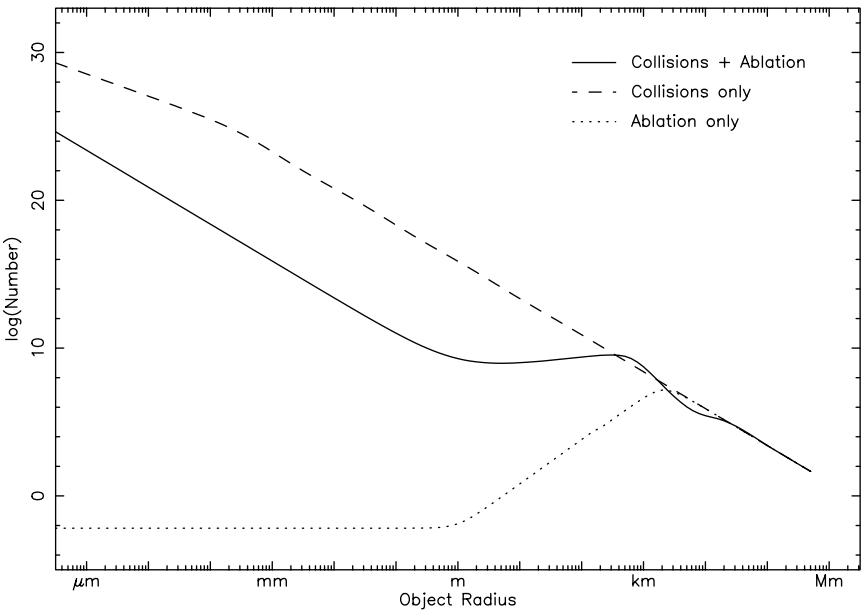

FIG. 3.-Distribution of particle sizes in a system with strong ablation by a relativistic particle wind. Sizes ranging from micron-sized dust up to $500 \mathrm{~km}$ radius planetesimals are considered. The number of particles is given per logarithmic size bin. Below $\sim 1 \mathrm{~km}$ in radius, objects are ablated by the pulsar wind faster than they are replenished by collisions of larger sized objects. The distribution of particle sizes produced by a standard collisional cascade is shown for comparison (dashed line), as is the case with ablation but no collisions (dotted line).

The particles emitted by the pulsar as it spins down, however, do carry their own positive angular momentum, which they would then impart on the dust grains.) Regardless of whether or not many submicron grains are present, their heating by the pulsar wind is very inefficient, as mentioned above. The larger planetesimals, on the other hand, are thick enough to absorb the pulsar's emission energy, and should reach temperatures comparable to the local blackbody temperature $(270 \mathrm{~K}$ at $2.5 \mathrm{AU})$. As in the pure ablation case, the majority of the warm surface area in the system continues to be contributed by these large $\sim$ kilometer-sized objects. The total emitting cross section for the model distribution is $4 \times 10^{19} \mathrm{~cm}^{2}$. This is a factor of 5 greater than with ablation alone (Fig. 3, dotted line), but still 3 orders of magnitude less than the area around the standard collisional cascade without ablation (dashed line). This area at $2.5 \mathrm{AU}$ corresponds to $L_{\mathrm{dust}} / L_{\mathrm{SD}}$ of just $2.3 \times 10^{-9}$, far below our observational limit.

Models in which disks steadily and smoothly grind away with time, however, fail to describe the variety of debris disks now observed with Spitzer. Just considering analytic models like those in Figure 3, one might conclude that all systems would grind down their asteroid belts in a fashion similar to our own, such that no old stars should have observable inner belts. This is in contrast to systems such as HD 69830, which has a bright disk of small dust grains orbiting at $\sim 0.5 \mathrm{AU}\left(L_{\text {dust }} / L_{\star} \sim 2 \times 10^{-4}\right.$; Beichman et al. 2005b). Despite the old age of this star ( 2 Gyr), the debris has an emitting surface area more than 1000 times greater than our asteroid belt's. As is clear from the collisional models, such a situation cannot persist for the lifetime of the star, but instead must be a reflection of some recent spike in activity. Based on the broad halo of small, short-lived dust grains blown out from Vega's debris disk, a large recent collisional event is also inferred in that system (Su et al. 2005). More generally, Rieke et al. (2005) find that while debris disk frequency declines as stars age, even old A stars can have significant emission due to stochastic collisional events, while Bryden et al. (2006) similarly find many bright disks among even older FGK stars. Overall, one can conclude that many, if not most, observed debris disks are observable only because an unusual event has recently increased their IR brightness. Such collisional events will also 
cause temporary enhancements of the IR emission from debris disks around pulsars. Although the model in Figure 3 is 4 orders of magnitude below the observational threshold presented in $\S 3$, modest improvements in the detection limit could detect the aftereffects of a large collisional event like those commonly seen around main-sequence stars, if one has recently occurred in the PSR B1257+12 system.

We conclude that, despite our new limits on IR excess, a $0.01 M_{\oplus}$ belt of $100 \mathrm{~km}$ sized asteroids and its collisionally produced dusty debris may still be present in this system. Whether or not this debris could be detected at far-IR wavelengths depends strongly on its recent collisional history.
This publication makes use of NASA/IPAC's InfraRed Science Archive (IRSA), which provides access to data from the 2MASS and IRAS all-sky surveys. The Spitzer Space Telescope is operated by the Jet Propulsion Laboratory, California Institute of Technology, under NASA contract 1407. Development of MIPS was funded by NASA through the Jet Propulsion Laboratory, subcontract 960785 . Some of the research described in this publication was carried out at the Jet Propulsion Laboratory, California Institute of Technology, under a contract with the National Aeronautics and Space Administration. We would like to thank Tom Kuiper for discussions on pulsar emission and an anonymous referee for helpful comments.
Abazajian, K., et al. 2005, AJ, 129, 1755

Aumann, H. H., et al. 1984, ApJ, 278, L23

Beckwith, S. V. W., Sargent, A. I., Chini, R. S., \& Guesten, R. 1990, AJ, 99, 924

Begelman, M. C., \& Li, Z.-Y. 1992, ApJ, 397, 187

Beichman, C. A., et al. 2005a, ApJ, 622, 1160 2005b, ApJ, 626, 1061

Bryden, G., et al. 2006, ApJ, 636, 1098

Cordes, J. M., \& Lazio, T. J. W. 2002, preprint (astro-ph/0207156)

Davis, D. R., Durda, D. D., Marzari, F., Campo Bagatin, A., \& Gil-Hutton, R.

2002, in Asteroids III, ed. W. F. Bottke, Jr., et al. (Tucson: Univ. Arizona Press), 545

Dermott, S. F., Durda, D. D., Grogan, K., \& Kehoe, T. J. J. 2002, in Asteroids III, ed. W. F. Bottke, Jr., et al. (Tucson: Univ. Arizona Press), 423

Dohnanyi, J. S. 1968, in IAU Symp. 33, Physics and Dynamics of Meteors, ed.

L. Kresak \& P. M. Millman (Dordrecht: Reidel), 486

Dole, H., et al. 2004, ApJS, 154, 93

Dominik, C., \& Decin, G. 2003, ApJ, 598, 626

Durda, D. D., \& Dermott, S. F. 1997, Icarus, 130, 140

Foster, R. S., \& Fischer, J. 1996, ApJ, 460, 902

Gaensler, B. M., \& Slane, P. O. 2006, ARA\&A, 44, 17

Gordon, K. D., et al. 2004, Proc. SPIE, 5487, 177 2005, PASP, 117, 503

Goździewski, K., Konacki, M., \& Wolszczan, A. 2005, ApJ, 619, 1084

Greaves, J. S., \& Holland, W. S. 2000, MNRAS, 316, L21

Gustafson, B. A. S. 1994, Annu. Rev. Earth Planet. Sci., 22, 553

Habing, H. J., et al. 2001, A\&A, 365, 545

Kennel, C. F., \& Coroniti, F. V. 1984, ApJ, 283, 710

Koch-Miramond, L., Haas, M., Pantin, E., Podsiadlowski, Ph., Naylor, T., \& Sauvage, M. 2002, A\&A, 387, 233

\section{REFERENCES}

Konacki, M., \& Wolszczan, A. 2003, ApJ, 591, L147

Lazio, T. J. W., \& Fischer, J. 2004, AJ, 128, 842

Lin, D. N. C., Woosley, S. E., \& Bodenheimer, P. H. 1991, Nature, 353, 827

Löhmer, O., Wolszczan, A., \& Wielebinski, R. 2004, A\&A, 425, 763

Love, S. G., \& Brownlee, D. E. 1993, Science, 262, 550

Malov, I. F. 2003, Astron. Lett., 29, 502

Marcy, G. W., \& Butler, R. P. 1995, BAAS, 27, 1379

Mayor, M., \& Queloz, D. 1995, Nature, 378, 355

McCaughrean, M. J., \& O’Dell, C. R. 1996, AJ, 111, 1977

Michel, F. C., \& Dessler, A. J. 1985, Science, 228, 1015

Miller, M. C., \& Hamilton, D. P. 2001, ApJ, 550, 863

Moshir, M., et al. 1990, IRAS Faint Source Catalogue (ver. 2.0; Greenbelt: NASA)

Phillips, J. A., \& Chandler, C. J. 1994, ApJ, 420, L83

Podsiadlowski, P., Pringle, J. E., \& Rees, M. J. 1991, Nature, 352, 783

Rieke, G. H., et al. 2005, ApJ, 620, 1010

Stern, S. A. 1996, A\&A, 310, 999

Stevens, I. R., Rees, M. J., \& Podsiadlowski, P. 1992, MNRAS, 254, 19P

Su, K., et al. 2005, ApJ, 628, 487

Tavani, M., \& Brookshaw, L. 1992, Nature, 356, 320

Taylor, J. H., \& Cordes, J. M. 1993, ApJ, 411, 674

van Buren, D., \& Terebey, S. 1993, in ASP Conf. Ser. 36, Planets Around

Pulsars (San Francisco: ASP), 327

Wolszczan, A. 1994, Science, 264, 538

Wolszczan, A., \& Frail, D. A. 1992, Nature, 355, 145

Wolszczan, A., Hoffman, I. M., Konacki, M., Anderson, S. B., \& Xilouris, K. M. 2000, ApJ, 540, L41 